\title{
High-Pressure Polymorphism of Acetylsalicylic Acid (Aspirin): Raman Spectroscopy
}

\author{
Ethan L. Crowell, Zbigniew A. Dreger, ${ }^{1}$ and Yogendra M. Gupta \\ Institute for Shock Physics and Department of Physics and Astronomy, \\ Washington State University, Pullman, WA 99164-2816, USA
}

${ }^{1}$ Corresponding author. E-mail address: dreger@wsu.edu; Tel.: 1-509-335-4233 (Z.A. Dreger) 


\begin{abstract}
Micro-Raman spectroscopy was used to elucidate the high-pressure polymorphic behavior of acetylsalicylic acid (ASA), an important pharmaceutical compound known as aspirin. Using a diamond anvil cell (DAC), single crystals of the two polymorphic phases of aspirin existing at ambient conditions (ASA-I and ASA-II) were compressed to $10 \mathrm{GPa}$. We found that ASA-I does not transform to ASA-II, but instead transforms to a new phase (ASA-III) above 2 GPa. It is demonstrated that this transformation primarily introduces structural changes in the bonding and arrangement of the acetyl groups and is reversible upon the release of pressure. In contrast, a less dense ASA-II shows no transition in the pressure range studied, though it appears to exhibit a disordered structure above $7 \mathrm{GPa}$. Our results suggest that ASA-III is the most stable polymorph of aspirin at high pressures.
\end{abstract}

Keywords: Polymorphism, high pressure, Raman spectroscopy, Aspirin 


\section{Introduction}

Organic molecules can often crystallize in more than one manner; a phenomenon referred to as polymorphism [1]. Different polymorphs may have differing physical and chemical properties, thereby affecting their performance and functionality. Hence, polymorphism is of great interest to the chemical industry in general, with a particular emphasis in pharmaceutical and explosives manufacturing. Typical methods for obtaining polymorphs include varying the crystallization techniques and their parameters. Another approach well suited for producing polymorphic phases is the application of high pressure. By changing the balance between various interactions (covalent, van der Waals, H- bonding, etc.), application of high pressure can change the interand intra-molecular arrangements and can, therefore, lead to the formation of new polymorphic phases. In addition, high-pressure experiments can provide new insight into the factors responsible for the packing and stability of different polymorphic phases.

Here we examine the effect of pressure on the stability and polymorphism of acetylsalicylic acid $\left(\mathrm{C}_{9} \mathrm{H}_{8} \mathrm{O}_{4}\right.$; ASA $)$ - an important analgesic and antiplatelet drug commonly known as aspirin. At ambient conditions, ASA is known to assume two polymorphic phases: ASA-I and ASA-II. ASA-I was thought to be the only form of aspirin for almost 40 years [2]. However, ASA-II was predicted computationally [3] in 2004 and shortly thereafter was experimentally observed and characterized [4]. This polymorph was initially found in intergrowth structures and transformed to ASA-I over time [5]. Only recently has clear evidence been provided for a pure, stable form of ASA-II [6].

In both polymorphs, the molecules are held together by van der Waals and H-bonded forces and they crystalize to the same monoclinic structure ( $\mathrm{P} 2{ }_{1} / \mathrm{c}$ space group), each having four molecules in the unit cell. There is also a large similarity between the molecular arrangements in 
the two structures $[5,6]$. Both structures contain centrosymmetric molecular dimers, held together by $\mathrm{O}-\mathrm{H} \cdots \mathrm{O}$ hydrogen bonds between their carboxyl $(\mathrm{COOH})$ groups (see, Figure $1 \mathrm{a}$, c). The dimers are arranged into two-dimensional layers in both cases. The primary difference between the two polymorphs lies in the way the layers are arranged and bonded. In the case of ASA-I, the molecules in direct contact across the layer boundary form the hydrogen-bonded, centrosymmetric $\mathrm{C}-\mathrm{H} \cdots \mathrm{O}$ dimers between the acetyl $\left(\mathrm{CH}_{3} \mathrm{CO}\right)$ groups. However, in the case of ASA-II the interlayer hydrogen bonds form the chain-like $\mathrm{C}-\mathrm{H} \cdots \mathrm{O}$ catemers (see Figure $1 \mathrm{~b}, \mathrm{~d}$ ). Computational studies have indicated that the energetics of the two structures are dominated by the competition between intramolecular conformations and intermolecular hydrogen bonding $[3,7]$. Specifically, it was shown that the catameric structure in ASA-II constrains the molecules to a higher-energy conformer than does the dimer structure in ASA-I. On the other hand, the presence of catameric chains between layers stabilizes the intermolecular interactions in ASA-II relative to ASA-I through hydrogen-bond cooperativity [7]. As a result, the two polymorphs are virtually isoenergetic, which could explain why intergrowths of the two packing motifs occur frequently. Although the computational work has provided some insight into the energetics of aspirin polymorphs, experimental results still lack evidence regarding the role of structural aspects in the polymorphic behavior.

In this work, we have used high pressure to provide further understanding of the factors responsible for the stability and polymorphic behavior of aspirin. There are several reasons for our approach. First, high pressure is well suited for altering the balance between intra- and intermolecular interactions, and therefore for shifting structural stability. Second, high pressure can tune the balance between the different types of hydrogen bonds that constitute the aspirin structure. In particular, the relatively weak interlayer $\mathrm{C}-\mathrm{H} \cdots \mathrm{O}$ bonding can be affected more 
than the stronger intralayer $\mathrm{O}-\mathrm{H} \cdots \mathrm{O}$ bonding. Since the former has different motifs in the two polymorphs, the application of high pressure can be used to examine their contribution to structural stability. Third, crystallization techniques have been the only methods employed to date for producing aspirin polymorphs, with ASA-I and ASA-II being the only polymorphs realized [4-6, 8-9]. Thus, high pressure can be used to explore the potential for producing other ASA polymorphs. Finally, the ASA-II unit cell is reported to have a slightly larger volume, being $101 \%$ that of ASA-I $[4,6]$. Hence, one might expect that ASA-II could transform to ASAI under the application of high pressure. This reasoning appears to have been followed by Bond et al [6]. However, their study did not go beyond 2.2 GPa and they used a mixture of ethanol and methanol as the pressure transmitting medium, both of which are known to dissolve aspirin. In their work, no transformation of ASA-II to ASA-I was observed.

In our work, we used high-pressure micro-Raman spectroscopy as a sensitive tool for detecting changes in bonding, interactions, and symmetries, and therefore in molecular and crystal structures. In particular, we wanted to address the following questions: first, how will high pressure affect molecular bonding and structural stability of the two aspirin polymorphs? Second, can high pressure generate additional polymorphs of aspirin? Third, can high pressure induce the transformation of ASA-II to ASA-I (or vice versa) at pressures above those previously attained? Fourth, is there a relationship between the response of the different potential polymorphs of aspirin?

\section{Experimental Methods}

Aspirin was purchased from ICN Biomedicals. ASA-I single crystals were grown by slow evaporation of a methanol solution at room temperature. To obtain ASA-II crystals, we followed 
the methods outlined by Chan et al [8]. ASA-I was dissolved in acetonitrile to produce a $\sim 0.6$ M solution. The solution was then held in an oil bath at approximately $75-85^{\circ} \mathrm{C}$ for one week. Finally, the flask with solution was plunged in an ice bath. This procedure provided good quality, small single crystals suitable for high-pressure experiments.

High pressure was generated using diamond anvil cell (DAC), utilizing brilliant cut anvils with $0.5 \mathrm{~mm}$ or $0.3 \mathrm{~mm}$ culets. In all cases cryogenically loaded argon was used as the pressure transmitting medium, to achieve hydrostatic conditions within the cell for the pressure range examined. Pressure was monitored via the ruby fluorescence method [10] with an accuracy of $\sim 0.05 \mathrm{GPa}$. Pressure-induced changes in the vibrational structure were determined using micro-Raman spectroscopy. The 532 line from a cw diode-pumped solid-state laser was employed for Raman excitation. A micro-Raman system (T64000) equipped with a microscope (Olympus BX-40) was used to provide spectra with resolution of $\sim 1 \mathrm{~cm}^{-1}$. All measurements were performed at room temperature. Details of the experimental setup for the Raman and ruby fluorescence measurements can be found elsewhere [11-13].

\section{Results and Discussion}

\subsection{Pressure Effects on ASA-I}

The measured vibrational modes of ASA-I single crystal at ambient and high pressure conditions are presented in Table 1. The modes in the region $1275 \mathrm{~cm}^{-1}$ to $1400 \mathrm{~cm}^{-1}$ were unresolved because of the strong Raman mode of diamond anvils centered at $1333 \mathrm{~cm}^{-1}$. Thus, the $\mathrm{OH}$ bending modes were not monitored in our experiments. All other previously reported Raman active modes [14] were resolved. The mode assignments at ambient pressure followed the work 
by Boczar et al, [14] which included both theoretical simulations and experimental results for Raman and IR active vibrations.

Pressure effects on the Raman spectrum of ASA-I, in selected frequency regions, are shown in Figure 2. Nearly all peaks gradually blue shifted as pressure was initially increased. At $\sim 2.3 \mathrm{GPa}$ there were several sudden changes in the spectrum as can be seen in Figures 3 and 4. These changes included (i) appearance and disappearance of modes, (ii) discontinuities in the Raman shift, and (iii) splitting of modes. The abruptness and extent of the changes indicate that a phase transition takes place at this pressure. All of these changes were reproducible in our experiments and occurred consistently at the same pressure.

From Table 1, we notice that the modes denoted as $v_{5}, v_{6}, v_{8}, v_{9}, v_{11}, v_{18}, v_{21}$, and $v_{36}$ can be used to monitor the response of the carboxyl group to pressure. Of these modes, only $v_{5}$, $v_{8}$, and $v_{36}$ displayed any changes at $2.3 \mathrm{GPa}$ (see Figure 3 ). The $\mathrm{C}=\mathrm{O}$ stretching mode $\left(v_{36}\right)$ redshifts discontinuously, at which point its slope increases (see dv/dp in Table 1), indicating softening of this mode. The slope of $\mathrm{v}_{8}$ nearly doubles, indicating that the $\mathrm{Ph}-\mathrm{COOH}$ bond becomes much more sensitive to pressures beyond $\sim 2 \mathrm{GPa}$. Although these changes may mark a change in the geometry of the carboxyl group, this change is likely not large. This conclusion is based on the behavior of the mode $v_{6}$, which corresponds to the stretching of $\mathrm{O}-\mathrm{H} \cdots \mathrm{O}$. This mode did not undergo any changes over the pressure range studied. This implies that the hydrogen bonding formed between the carboxyl groups remains intact beyond $2 \mathrm{GPa}$. Consequently, one would not expect large changes in the arrangement of the carboxyl group.

Unlike the carboxyl group, the acetyl group appears to undergo considerable changes at $\sim 2 \mathrm{GPa}$ (see pressure shifts in Figures 3 and 4 ). The pressure coefficient of mode $v_{30}$ increases 
abruptly, indicating that the bonding within the acetyl group becomes softer at this pressure. However, the opposite behavior of modes $v_{12}$ and $v_{31}$ implies that the $\mathrm{C}-\mathrm{O}$ bond connecting the acetyl group to the benzene ring actually becomes stiffer. Furthermore, the modes $v_{30}, v_{31}$, and $v_{38}$ all exhibit a discontinuous redshift, indicating a change in the geometry of the acetyl group. A much different effect is seen in the bending mode of $\mathrm{CH}_{3}\left(v_{33}\right)$. Initially, the frequency of this mode barely changes with pressure. However, at $\sim 2.3 \mathrm{GPa}$ its frequency jumps up about $8 \mathrm{~cm}^{-1}$ and then demonstrates a substantial blue shift with increasing pressure. All of these discontinuities are likely due to a change in the arrangement of the acetyl group.

In addition to discontinuities and changes in the slope of the Raman shifts, there are also changes in the number of active modes. For example, the torsion mode of $\mathrm{CH}_{3}\left(\mathrm{v}_{7}\right)$ and some weak stretching modes of $\mathrm{CH}_{\text {methyl }}\left(v_{39}\right.$ and $\left.v_{41}\right)$ disappear above $\sim 2 \mathrm{GPa}$. On the other hand, modes $v_{16}$ and $v_{37}$ split into the doublets $\left(v_{16}, v^{3}\right)$ and $\left(v_{37}, v^{4}\right)$. These features further confirm the occurrence of a phase transition above $2 \mathrm{GPa}$. It is interesting to note that $v^{3}$ and $v^{4}$ are located approximately where one would expect to observe the IR active modes [14]. We also notice that the $\mathrm{CH}$ stretching modes, both on the methyl group and the ring, are the most affected at the transition by a reduction in the number of modes from six to three. However, since new modes show up in other regions of the spectrum, it is not clear if the molecular symmetry was affected at the transition.

Further increase of pressure to $10 \mathrm{GPa}$ did not show any noticeable changes in the spectra, demonstrating that the new phase is stable over a broad pressure range. Successive release of pressure revealed that the Raman spectrum upon release is identical to that at 1 atm, indicating that the high pressure phase was not recovered at ambient conditions. 


\subsection{Pressure Effect on ASA-II}

In Table 2 we present the vibrational modes of ASA-II single crystal at ambient and high pressure conditions. To our knowledge, this is the first report presenting the Raman spectrum of ASA-II. We use the symbol $f$ to denote the ASA-II Raman modes to distinguish them from the ASA-I modes (denoted as $v$ ). The ASA-II modes present at ambient conditions are denoted with subscripts, whereas the peaks/shoulders that appeared at high pressures are denoted with superscripts. There is a clear correspondence between the ASA-II and ASA-I modes at ambient conditions (compare Tables $1 \& 2$ and Figures $2 \& 5$ ). As such, we used the aforementioned work of Boczar et al [14] as a tentative assignment for these modes. The number of Raman peaks observed experimentally for ASA-II is somewhat larger than that for ASA-I. However, it is still much lower than the number predicted theoretically [14].

In Figure 5, we show the Raman spectra of ASA-II at several pressures. The spectra are presented over the same frequency ranges as presented for ASA-I. The pressure shifts in the region $0-600 \mathrm{~cm}^{-1}$ are shown in Figure 6. It is important to note that the Raman shifts were gradual with increasing pressure. The lack of discontinuities in both the position and the pressure coefficient allowed us to fit each mode with a single equation. The pressure coefficients of the Raman modes' shifts can be found in Table 2 .

In addition to the gradual shift of the Raman peaks, we also observed some new features in the spectra with increased pressure. First, some weak intensity peaks disappeared at higher pressures (modes $f_{7}, f_{9}$, and $f_{38}$ in Table 2). However, the intensity of these peaks decreased gradually over the extended range of pressures. Hence, it is likely that these modes remained Raman active throughout the pressure range and simply became too weak to be resolved. In addition to this, a few Raman modes were observed to appear at high pressures. There seems to 
be a new mode splitting off $f_{5}\left(106 \mathrm{~cm}^{-1}\right)$ at around $2.5 \mathrm{GPa}$. However, it is likely that this peak is not actually new, but was covered by the more intense peaks in the densely populated low frequency region. Also, there is the apparent development of shoulders on some Raman peaks at various pressures. In particular, the shoulders appear around $292 \mathrm{~cm}^{-1}$ at $\sim 9 \mathrm{GPa}, 1201 \mathrm{~cm}^{-1}$ at $\sim 3.4 \mathrm{GPa}$, and $2956 \mathrm{~cm}^{-1}$ at $\sim 5 \mathrm{GPa}$ (see Figure 5). Finally, above $7 \mathrm{GPa}$ there was considerable increase in the width and some decrease in intensity of selected Raman peaks.

There are three potential causes for the above indicated behavior: i) a phase transformation, ii) nonhydrostatic effects, and/or iii) structural disorder. There are two reasons why it is unlikely that ASA-II changed phase. First, in the case of a phase transition one would expect to see discontinuities in the Raman shifts at some pressures. However, as mentioned above, all the observed shifts were gradual. Second, the effects appeared at a variety of pressures ranging from $\sim 3 \mathrm{GPa}$ to $\sim 9 \mathrm{GPa}$, whereas one would expect a phase transition to occur within a narrow range of pressures. In the case of nonhydrostaticity we would expect (i) broadening of most Raman peaks and (ii) changes in the ruby lines (width, separation). Neither of these effects was observed. On the other hand, only the selected Raman modes show any effects. Hence, the broadening and appearance of shoulders on some peaks can imply that this behavior is likely due to some distribution in molecular arrangement and distortion of the molecular bonding, instead of pressure gradients within the cell. The physical reason for the structural disorder of ASA-II at high pressures is not presently understood. However, it should be pointed out that these effects were reversible upon pressure release. Furthermore, these effects were not observed for ASA-I. 


\section{Relations between High Pressure Polymorphs of Aspirin}

Next, we discuss possible factors responsible for the differences in the behavior of the ASA polymorphs under high pressure. In particular, we focus on structural aspects.

ASA-I and ASA-II showed marked differences in their behavior in the pressure range below $\sim 2 \mathrm{GPa}$. Many of the modes corresponding to the acetyl group (see modes $v_{6}, v_{10}, v_{12}, v_{24}, v_{37}$, and $v_{38}$ in Table 1 ) were significantly softer in ASA-I than their counterparts in ASA-II. The hydrogen bond cooperativity resulting from the catamers in ASA-II provides a relatively rigid bonding scheme, whereas the dimers in ASA-I give the acetyl groups more freedom to relax [7]. Hence, it is likely that the stiffness of these modes in ASA-II is due to the presence of the catameric chains. The carboxyl group, on the other hand, showed no significant difference in its behavior for the two phases. Because the carboxyl groups are nearly identical in the two phases, this similarity in behavior is consistent with expectations.

The most striking contrast between ASA-I and ASA-II is seen at $~ 2$ GPa. ASA-I undergoes a phase transition at this pressure, while the ASA-II spectrum showed no changes. Thus, high pressure appears to lift the energetic "degeneracy" [7] of the two phases. Figure 7 presents the spectra of ASA-I measured at $1.9 \mathrm{GPa}$ and $2.3 \mathrm{GPa}$, and the ASA-II spectrum measured at 2.0 GPa. By comparing the spectra, we rule out the possibility that an ASA-I to ASA-II phase transition is the result of this shift in the relative energetics. Consequently, we demonstrate that ASA-I transforms to another polymorph of aspirin (ASA-III) above 2 GPa.

It is interesting to note that no transition from ASA-I to ASA-II was observed. This may be understood by examining the structural differences between ASA-I and ASA-II in the context of pressure-induced changes in ASA-I. As shown in Figure 8a, there are two conformations that are assumed by ASA molecules in the crystal structure; we denote them as A and B. Due to 
differences in the orientation of the acetyl group, the two conformers are not equivalent with respect to translation and rotation of the molecule as a whole. In both ASA-I and ASA-II, the carboxyl dimers involve conformers of opposite type; that is, type-A conformers are bonded to type-B conformers (see graphs in Figure 8b). In ASA-I, the acetyl dimers also consist of conformers of opposite type, whereas in ASA-II they involve bonds between conformers of like type (see the arrangement of molecules in rectangles in Figure 8b). In addition to these differences, the acetyl dimers consist of parallel molecules in ASA-I, which is not the case in ASA-II (see Figure 1 b, d).

Due to these differences, one may conceive of two potential paths for a transition between ASA- I and ASA-II. The first path would require a two-step modification in the crystal: (i) a $180^{\circ}$ rotation of the acetyl group around the $\mathrm{C}-\mathrm{O}$ bond to transform conformers of type-A to type-B (and vice versa), and (ii) a rotation of these molecules about the c-axis. This path may be understood by comparing the two regions enclosed by white rectangles in Figure $8 \mathrm{~b}$. The second path would require entire planes of the carboxyl dimers to shift along the c-axis. As seen in Figure 8b, such a translation of about one half of a unit cell would produce the acetyl bonding scheme of ASA-II in ASA-I. In the case of both paths, the bonding between carboxyl groups must remain unchanged, as the carboxyl bonding scheme is identical in the two polymorphs. From these considerations, it is clear that the changes required in both paths to accomplish a transition from ASA-I to ASA-II are quite complex, requiring more than a mere rearrangement of the acetyl groups. On the other hand, the observed changes in the Raman spectra indicated that only the acetyl groups are affected at the transition at $2 \mathrm{GPa}$. Hence, this might explain why we do not observe a transition from ASA-I to ASA-II under high pressure. 
Finally, we discuss the relative stabilities between ASA-II and ASA-III under high pressure. Our data reveal that neither of the two polymorphs undergoes a phase transition in the pressure range 2-10 GPa. However, ASA-II exhibited a disordered structure at high pressures whereas ASA-III displayed no such behavior. In addition to this, it is interesting to note that modes $v_{6}, v_{10}, v_{37}$, and $v_{38}$ became much stiffer after the ASA-I to ASA-III transition. In fact, they are stiffer in ASA-III than they are in ASA-II. As the stiffness of these modes in ASA-II is primarily due to the catameric chains, our data suggest that the acetyl catamers are more resilient than the acetyl dimers at high pressure. Therefore, one possibility is that the ASA-I to ASA-III phase transition is the result of the acetyl bonding scheme changing to a catamer-like scheme; high pressure single-crystal x-ray diffraction experiments are required to examine this conjecture. The above considerations indicate that ASA-III is more durable at high pressure than ASA-II. Future work will include developing methods for quenching ASA-III to ambient conditions, allowing its bio-functionality to be investigated.

\section{Conclusions}

Using Raman spectroscopy the stability of two polymorphic forms of aspirin (ASA-I and ASAII) was examined to $10 \mathrm{GPa}$. ASA-I was found to undergo a phase transition to a new polymorph (ASA-III) at $2 \mathrm{GPa}$. The ASA-I to ASA-III phase transition primarily involved changes in the acetyl groups' bonding, without affecting the bonding of the carboxyl groups. In contrast to ASA-I, ASA-II did not undergo any phase transition in the pressure range studied; however it exhibited a disordered structure above $7 \mathrm{GPa}$. The disordered structure observed in ASA-II at high pressures and the rigidity of the acetyl groups observed in ASA-III suggest that ASA-III is the most stable phase of aspirin at high pressure. 


\section{Acknowledgements}

This work was supported by the DOE-NNSA Grant \# DE-NA0000970 and ONR Grant \# N000014-12-1-0555. 


\section{References}

[1] J. Bernstein, Polymorphism of Molecular Crystals, Oxford University Press, New York, 2002.

[2] P.J. Wheatley, The crystal and molecular structure of aspirin, J. Chem. Soc. (1964) 60366048.

[3] C. Ouvrard, S.L. Price, Toward crystal structure prediction for conformationally flexible molecules: the headaches illustrated by aspirin, Cryst. Growth Des. 4, (2004) 1119-1127.

[4] P. Vishweshwar, J.A. McMahon, M. Oliveira, M.L. Peterson, M.J. Zaworotko, The predictably elusive form II of aspirin, J. Am. Chem. Soc. 127 (2005) 16802-16803.

[5] A.D. Bond, R. Boese, G.R. Desiraju, On the polymorphism of aspirin: crystalline aspirin intergrowths of two "polymorphic" domains, Angew. Chem. Int. Ed. 46 (2007) 618-622.

[6] A.D. Bond, K.A. Solanko, S. Parsons, S. Redder, R. Boese, Single crystals of aspirin form II: crystallization and stability, Cryst. Eng. Comm. 13 (2011) 399-401.

[7] S. Wen, G.J.O. Beran, Accidental degeneracy in crystalline aspirin: new insight from highlevel ab initio calculations, Cryst. Growth Des. 12 (2012) 2169-2172.

[8] E.J. Chan, T.R. Welberry, A.P. Heerdege, D.J. Goossens, Diffuse scattering study of aspirin forms (i) and (II), Acta Cryst. B 66 (2010) 696-707.

[9] B.B. Koleva, Polymorphs of aspirin - solid-state IR-LD spectroscopic and quantitative determination in solid mixture, J. Mol. Struct. 800 (2006) 23-27.

[10] G.J. Piermarini, S. Block, J. D. Barnett, R.A. Forman, Calibration of the pressure dependence of the R1 ruby fluorescence line to 195 kbar, J. Appl. Phys. 46 (1975) 27742780. 
[11] Z.A. Dreger, Y. M. Gupta, High Pressure Raman Spectroscopy of Single Crystals of Hexahydro-1,3,5-trinitro-1,3,5-triazine (RDX), J. Phys. Chem. A 111 (2007) 3893-3903.

[12] Z.A. Dreger, Y. M. Gupta, Raman Spectroscopy of High-Pressure-High-Temperature Polymorph of Hexahydro-1,3,5-trinitro-1,3,5-triazine ( $\varepsilon$-RDX), J. Phys. Chem. A 114 (2010) 7038-7047.

[13] Z.A. Dreger, Y. M. Gupta, High Pressure-High Temperature Polymorphism and Decomposition of Pentaerythritol Tetranitrate (PETN), J. Phys. Chem. A 117 (2013) 53065313.

[14] M. Boczar, M.J. Wójcik, K. Szczeponek, D. Jamróz, A. Zięba, B. Kawałek, Theoretical modeling of infrared spectra of aspirin and its deuterated derivative, Chem. Phys. 286 (2003) 63-79. 


\section{Figure Captions}

Figure 1. Aspirin crystal structures at ambient conditions: ASA-I (a and b) and ASA-II (c and d). (a) and (c): Projections of ASA-I and ASA-II structures along the b axis; the layers formed by the carboxyl dimers are highlighted. (b) and (d): different motifs of intermolecular hydrogen bonding between acetyl groups for ASA-I and ASA-II. Molecules are represented by the stick model. Legend: Carbon, grey; Oxygen, red; Hydrogen, white. Hydrogen bonding is denoted by the dashed blue lines.

Figure 2. Raman spectra of ASA-I at several pressures over different frequency ranges: a) 20$1100 \mathrm{~cm}^{-1}$, b) $\mathrm{CH}$ streching vibrations. Pressures listed next to each spectra are in GPa.

Figure 3. Pressure shifts of Raman modes of ASA-I: a) $50-600 \mathrm{~cm}^{-1}$ and b) $1100-1300 \mathrm{~cm}^{-1}$. The onset of the phase change is marked by the dashed line. The modes discussed in the text are denoted as in Table 1.

Figure 4. Pressure shifts of $\mathrm{CH}$ stretching modes of ASA-I. The onset of the phase transition is marked by the dashed line. The modes discussed in the text are denoted as in Table 1.

Figure 5. Raman spectra of ASA-II at several pressures in the a) $20-1100 \mathrm{~cm}^{-1}$, b) $\mathrm{CH}$ stretching modes. The modes discussed in the text are denoted as in Table 2.

Figure 6. Pressure shifts of Raman modes of ASA-I in the frequency region $25-650 \mathrm{~cm}^{-1}$. The modes discussed in the text are denoted as in Table 2.

Figure 7. Raman spectra of three aspirin polymorps at similar pressures: ASA-I at 1.9 GPa, ASA-II at 2.0 GPa, and ASA-III at 2.3 GPa. a) spectra in the range $50-1150 \mathrm{~cm}^{-1}$, b) spectra in the range $1400-1800 \mathrm{~cm}^{-1}$, and c) spectra in the range $2900-3150 \mathrm{~cm}^{-1}$. 
Figure 8. a) The two molecular conformations of aspirin. b) Projections of ASA-I and ASA-II structures along the $\mathrm{b}$ axis; type-A conformers are highlighted to distinguish them from type-B. The two regions enclosed in white rectangles highlight the differences in (i) conformer type and (ii) molecular orientations involved in the acetyl bonding schemes of the two phases. 
Figure 1, Crowell et al.
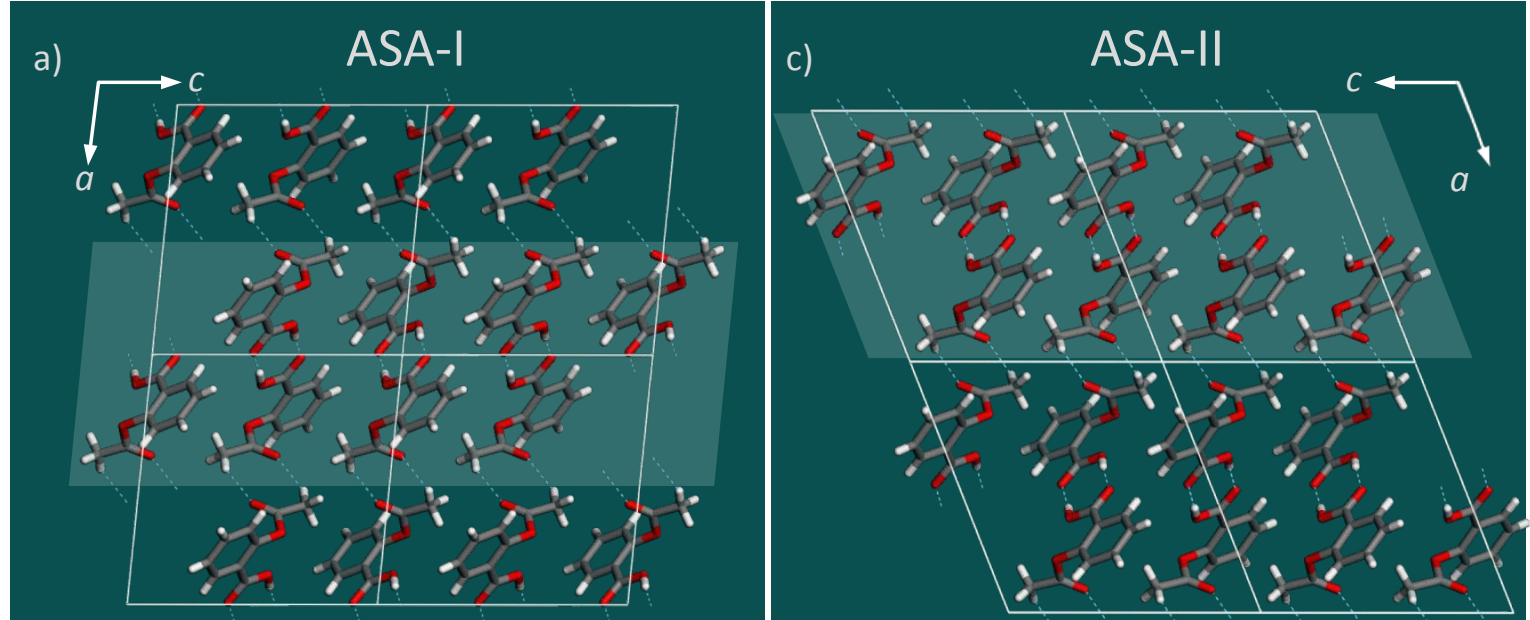

b)

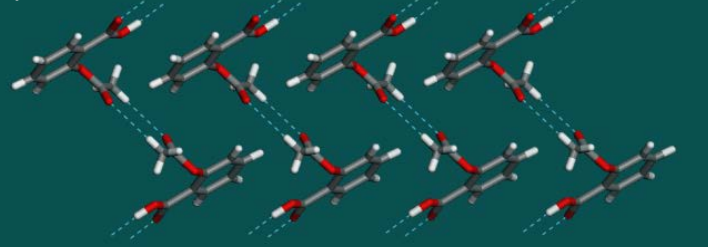

d)

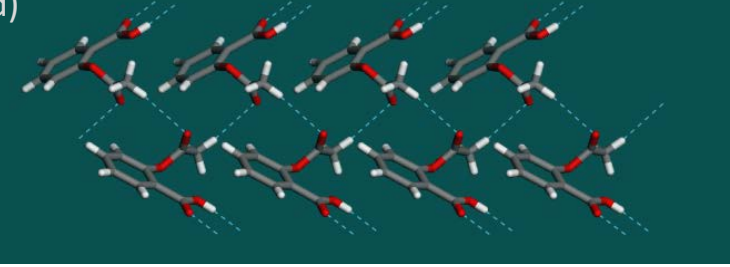


Figure 2, Crowell et al.
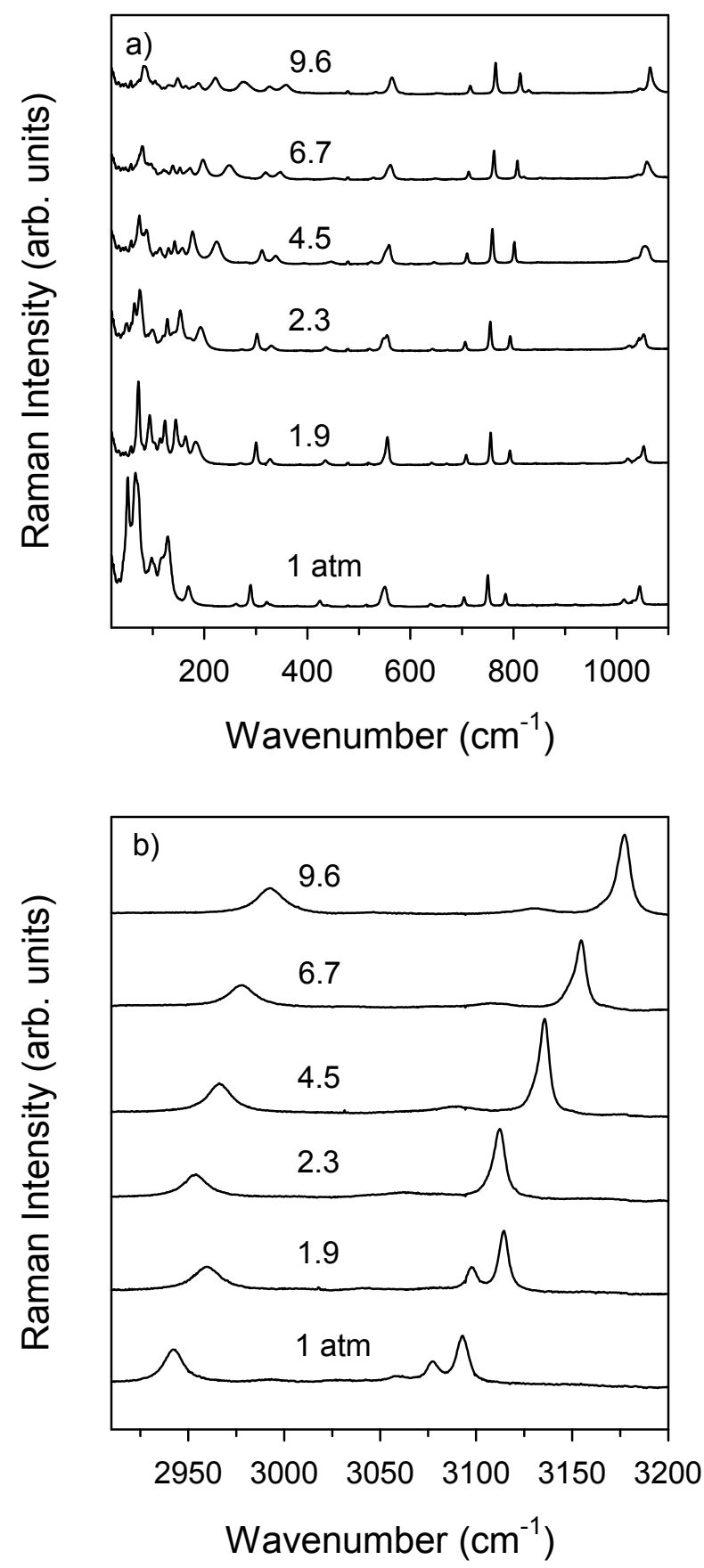
Figure 3, Crowell et al.
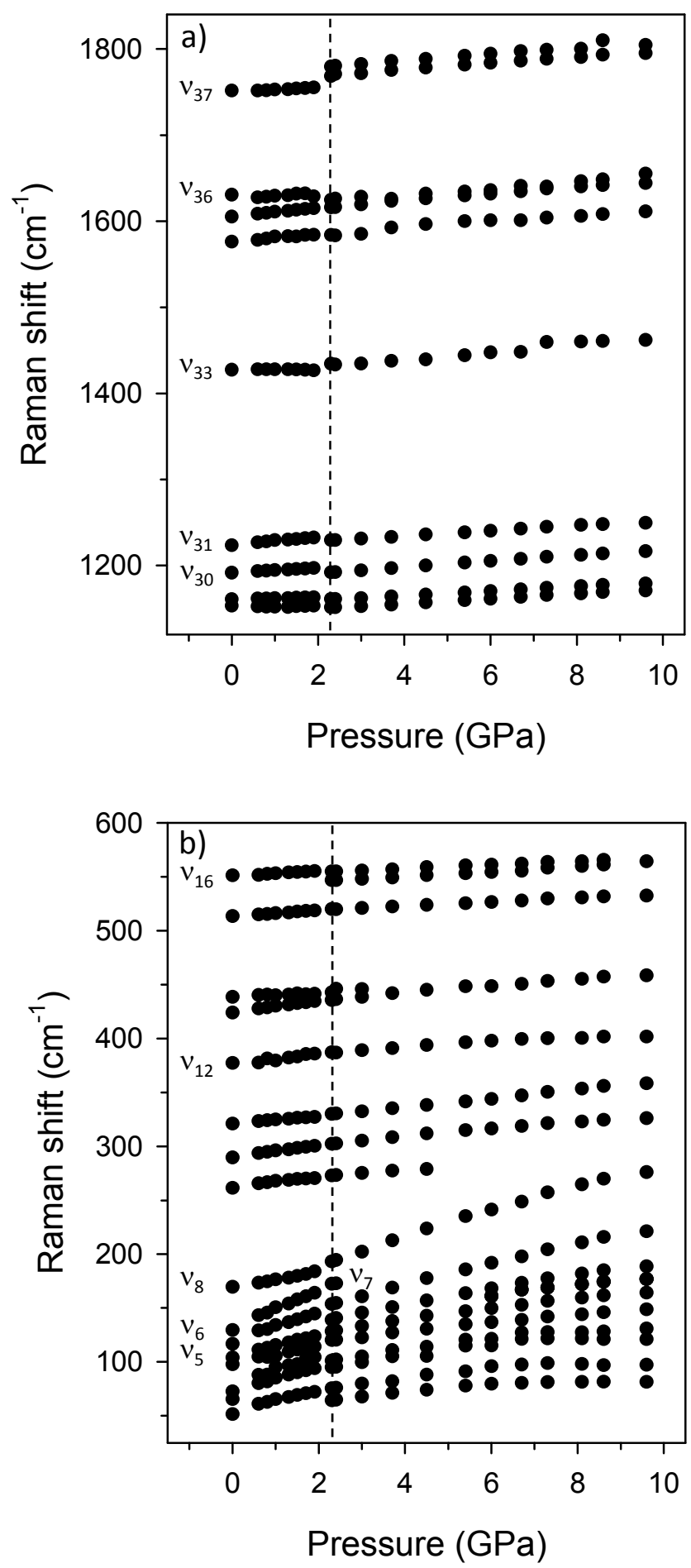
Figure 4, Crowell et al.

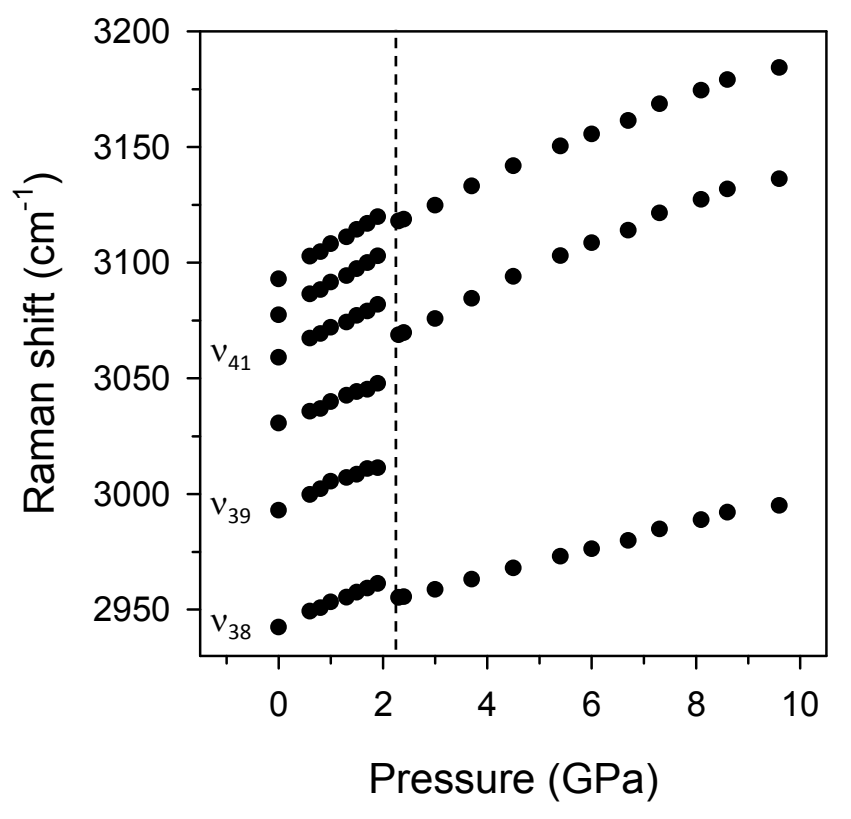


Figure 5, Crowell et al.
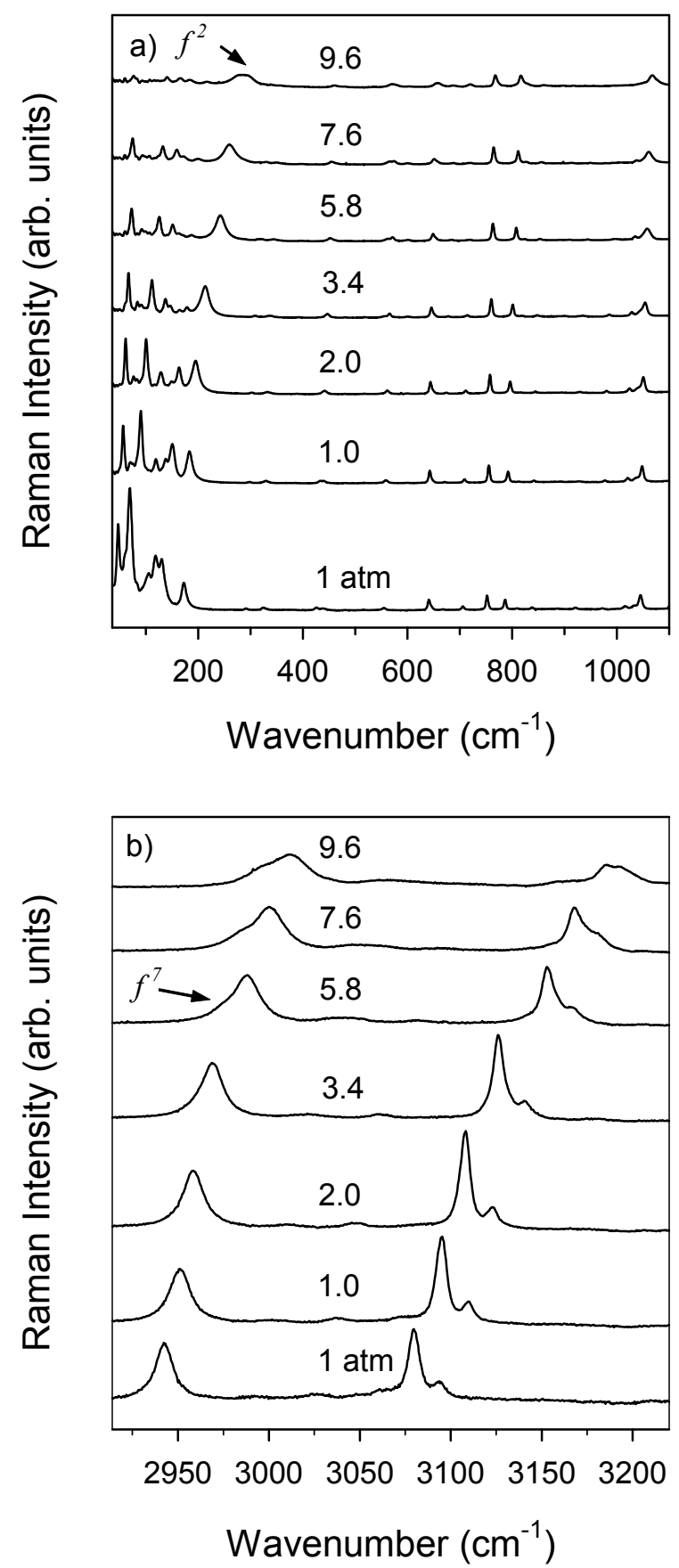
Figure 6, Crowell et al.

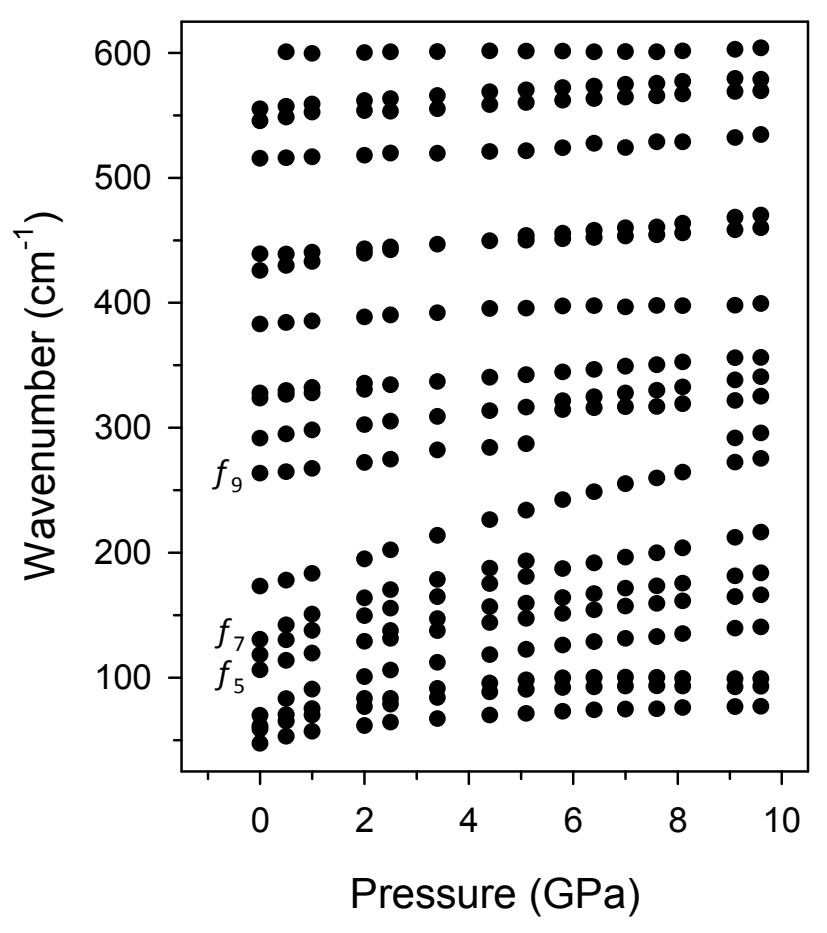


Figure 7, Crowell et al.
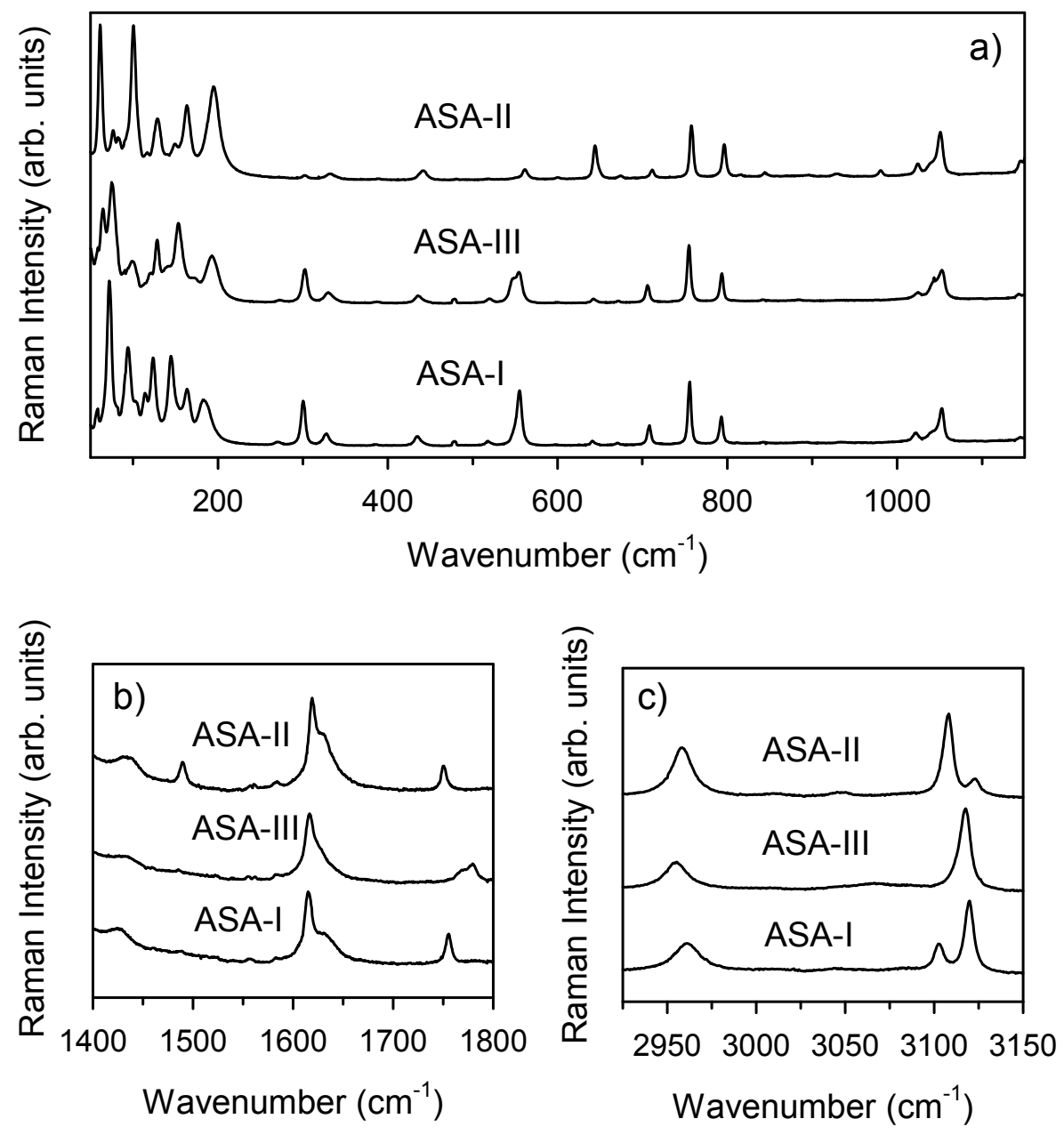
Figure 8, Crowell et al.

a)

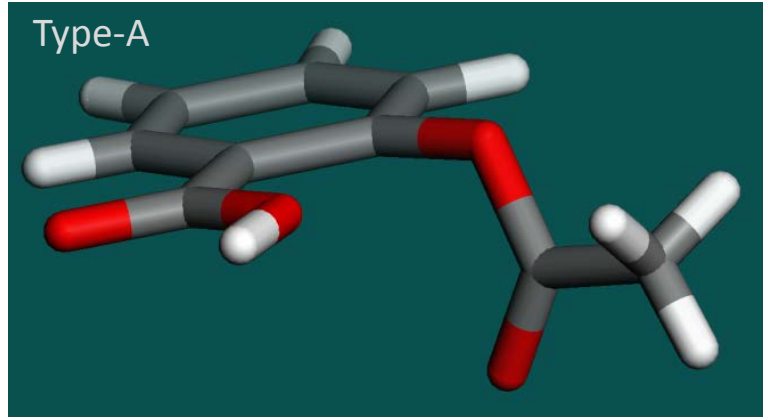

b)

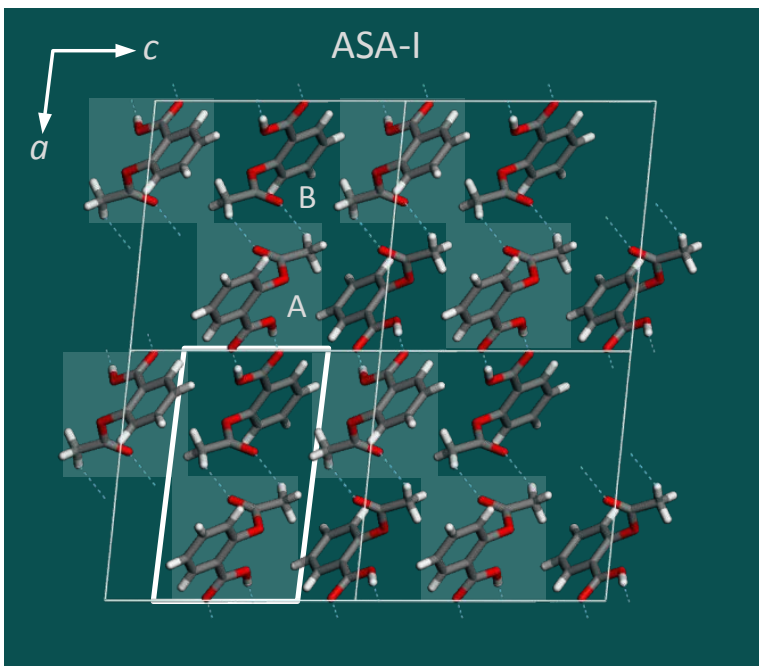

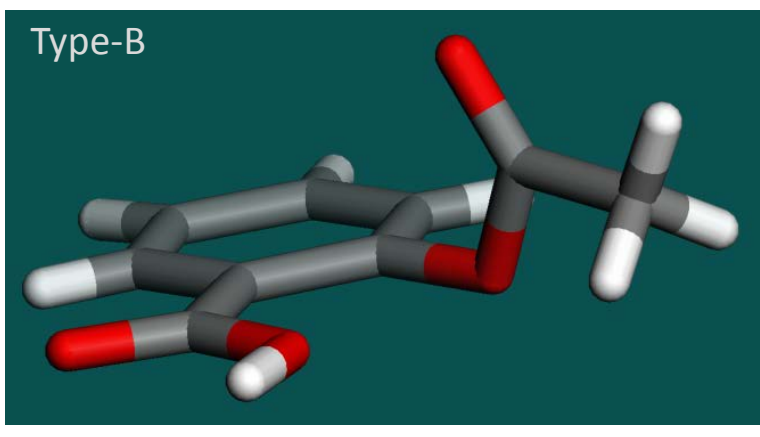

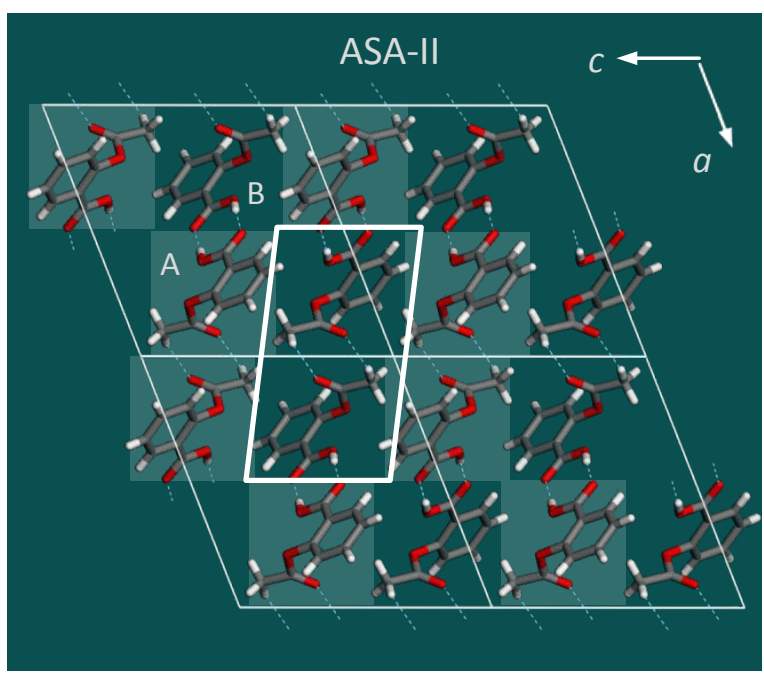


Graphical Abstract
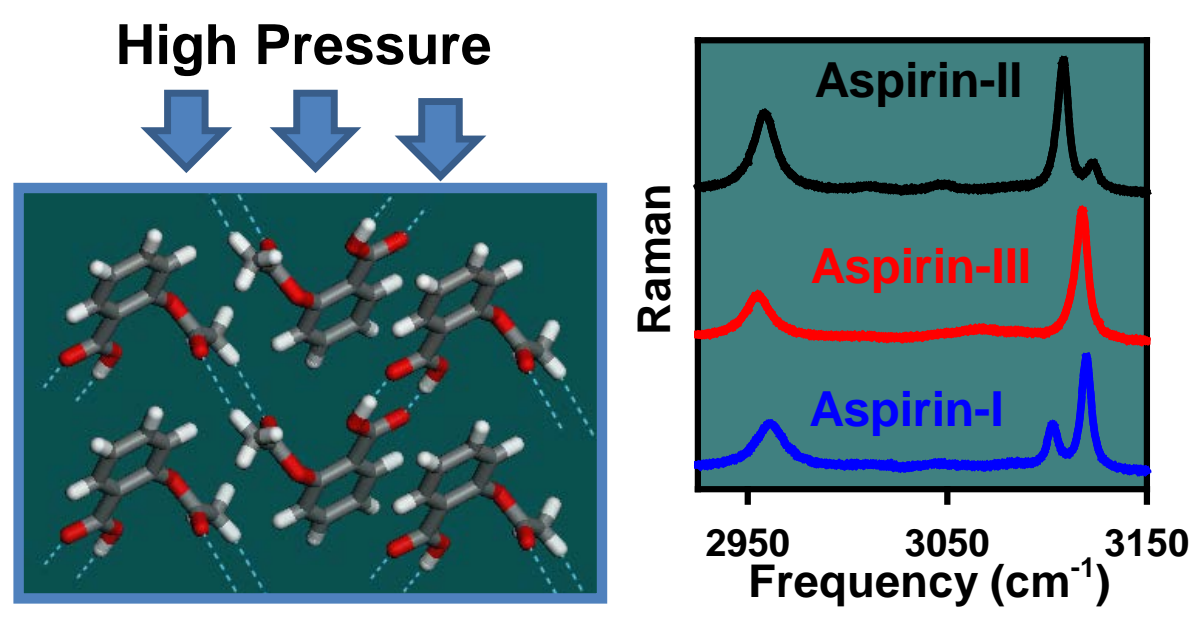
TABLE 1: Pressure effects on Raman modes of ASA-I single crystal

\begin{tabular}{|c|c|c|c|c|c|c|c|}
\hline \multirow[b]{3}{*}{$\operatorname{mode}^{a}$} & \multicolumn{4}{|c|}{$1 \mathrm{~atm}$} & \multirow{2}{*}{\multicolumn{3}{|c|}{$\begin{array}{c}\text { high pressure } \\
\mathrm{d} v / \mathrm{dp} \\
\left(\mathrm{cm}^{-1} \mathrm{GPa}^{-1}\right)\end{array}$}} \\
\hline & \multicolumn{2}{|c|}{ frequency $\left(\mathrm{cm}^{-1}\right)$} & \multirow[b]{2}{*}{ assignment $^{c}$} & \multirow{2}{*}{$\begin{array}{c}\begin{array}{c}\mathrm{d} v / \mathrm{dp}^{d} \\
\left(\mathrm{~cm}^{-1} \mathrm{GPa}^{-1}\right)\end{array} \\
\mathrm{a} \\
\end{array}$} & & & \\
\hline & $\begin{array}{l}\text { expt, this } \\
\text { work }\end{array}$ & $\operatorname{expt}^{b}$ & & & freq $^{e}$ & $\mathrm{a}$ & $\mathrm{b}$ \\
\hline$v^{1}$ & & & & & 64 & & \\
\hline$v_{1}$ & 51 & & & 10.4 & 75 & 10.5 & -0.6 \\
\hline$v_{2}$ & 65 & & & 14.2 & 95 & 10.3 & -0.5 \\
\hline$v_{3}$ & 72 & & & 15.9 & 101 & 9.6 & -0.5 \\
\hline$v_{4}$ & 98 & & & 8.6 & 120 & 4.0 & \\
\hline$v_{5}$ & 104 & 103 & $\mathrm{CH}_{3}$ tor $+\mathrm{Ph}-\mathrm{COOH}$ tor & 10.2 & 128 & 8.0 & -0.3 \\
\hline$v^{2}$ & & & & & 139 & 10.2 & -0.3 \\
\hline$v_{6}$ & 117 & 119 & $\mathrm{O} \cdots \mathrm{O}$ st $+\mathrm{CH}_{3}$ tor & 14.0 & 154 & 9.6 & \\
\hline$v_{7}$ & 130 & 131 & $\mathrm{CH}_{3}$ tor & 17.7 & & & \\
\hline$v_{8}$ & 170 & 171 & $\mathrm{Ph}-\mathrm{COOH} \mathrm{b}$ & 7.4 & 193 & & \\
\hline$v_{9}$ & 261 & 263 & $\mathrm{Ph}-\mathrm{COOH} \mathrm{b}$ & 4.7 & 273 & 2.7 & \\
\hline$v_{10}$ & 290 & 291 & $\mathrm{Ph}-\mathrm{OCOCH}_{3} \mathrm{~b}$ & 5.6 & 302 & 3.4 & \\
\hline$v_{11}$ & 321 & 323 & $\mathrm{C}-\mathrm{C}(\mathrm{COOH})-\mathrm{C} \mathrm{b}$ & 3.2 & 330 & 4.0 & \\
\hline$v_{12}$ & 377 & 381 & $\mathrm{OCOCH}_{3}$ rock. $\mathrm{b}+\mathrm{COOH}$ rock. $b$ & 4.8 & 387 & 5.1 & -0.3 \\
\hline$v_{13}$ & 424 & 425 & $\mathrm{O}-\mathrm{CO}-\mathrm{CH}_{3}$ sci b $+\mathrm{CC}_{\text {rings }} \mathrm{b}$ & 5.6 & 436 & 3.2 & \\
\hline$v_{14}$ & 439 & 443 & $\mathrm{OCOCH}_{3} \mathrm{~b}+\mathrm{CC}_{\text {rings }} \mathrm{b}$ & 1.4 & 443 & & \\
\hline$v_{15}$ & 514 & & & 2.8 & 520 & 1.8 & \\
\hline$v^{3}$ & & & & & 547 & 2.3 & \\
\hline$v_{16}$ & 551 & 551 & $\mathrm{CC}_{\text {rings }} \mathrm{b}+\mathrm{CO}-\mathrm{CH}_{3}$ sci $\mathrm{b}$ & 2.2 & 555 & 1.5 & \\
\hline$v_{17}$ & 640 & & & 1.1 & 643 & 1.5 & \\
\hline$v_{18}$ & 665 & 666 & $\mathrm{CC}_{\text {rings }} \mathrm{b}+\mathrm{O}-\mathrm{C}=\mathrm{O} \mathrm{b}+\mathrm{COOH}$ sci $\mathrm{b}$ & 3.1 & 672 & 2.0 & \\
\hline$v_{19}$ & 704 & 705 & $\mathrm{CH}_{\text {rings }} \mathrm{b}+\mathrm{CC}_{\text {rings }} \mathrm{b}$ & 2.1 & 706 & 1.5 & \\
\hline$v_{20}$ & 750 & 751 & $\mathrm{CH}_{\text {rings }} \mathrm{b}$ & 2.8 & 755 & 1.5 & \\
\hline$v_{21}$ & 784 & 785 & $\mathrm{CH}_{\text {rings }} \mathrm{b}+\mathrm{COOH} \mathrm{b}$ & 4.4 & 793 & 2.8 & \\
\hline$v_{22}$ & 837 & 837 & $\mathrm{CH}_{\text {rings }} \mathrm{b}$ & & 842 & & \\
\hline$v_{23}$ & 920 & 920 & $\mathrm{CC}_{\text {rings }} \mathrm{b}+\mathrm{O}-\mathrm{CO}-\mathrm{CH}_{3} \mathrm{~b}$ & & & & \\
\hline$v_{24}$ & 1014 & 1015 & $\mathrm{O}-\mathrm{CO}-\mathrm{CH}_{3} \mathrm{~b}+\mathrm{CH}_{3} \mathrm{~b}$ & 3.9 & 1025 & 7.5 & -0.4 \\
\hline$v_{25}$ & 1034 & & & 4.3 & 1044 & 3.1 & \\
\hline$v_{26}$ & 1045 & 1045 & sym.rings breathing $+\mathrm{CH}_{\text {rings }} \mathrm{b}$ & 4.1 & 1053 & 2.4 & \\
\hline$v_{27}$ & 1136 & 1137 & $\mathrm{CH}_{\text {rings }} \mathrm{b}$ & 4.3 & 1143 & 4.4 & -0.2 \\
\hline$v_{28}$ & 1153 & 1154 & $\mathrm{CH}_{\text {rings }} \mathrm{b}$ & 0.1 & 1151 & 2.8 & \\
\hline$v_{29}$ & 1161 & & & 1.2 & 1161 & 2.6 & \\
\hline$v_{30}$ & 1191 & 1191 & $\mathrm{O}-\mathrm{CO}-\mathrm{CH}_{3} \mathrm{st}+\mathrm{CH}_{3} \mathrm{~b}$ & 2.9 & 1192 & 3.4 & \\
\hline$v_{31}$ & 1223 & 1223 & $\mathrm{Ph}-\mathrm{OCOCH}_{3} \mathrm{st}+\mathrm{CH}_{\text {rings }} \mathrm{b}$ & 4.6 & 1229 & 2.9 & \\
\hline$v_{32}$ & 1258 & 1258 & $\mathrm{CH}_{\text {rings }} \mathrm{b}$ & & & & \\
\hline$v_{33}$ & 1427 & 1429 & $\mathrm{CH}_{3} \mathrm{~b}$ & -0.3 & 1434 & 4.4 & \\
\hline
\end{tabular}




$\begin{array}{llllllll}v_{34} & 1576 & 1576 & \mathrm{CC}_{\text {rings }} \text { st } & 4.4 & 1584 & 7.1 & 0.07 \\ v_{35} & 1605 & 1606 & \mathrm{CC}_{\text {rings }} \mathrm{st} & 5.2 & 1616 & 5.6 & -0.1 \\ v_{36} & 1631 & 1628 & \mathrm{C}=\mathrm{O} \text { carbox. st }+\mathrm{OH} \mathrm{b} & 0.8 & 1625 & 3.9 & \\ v_{37} & 1752 & 1752 & \mathrm{C}=\text { O ester st } & 1.1 & 1768 & 3.6 & \\ v^{4} & & & & & 1779 & 4.8 & -0.1 \\ v_{38} & 2942 & 2942 & \mathrm{CH}_{\text {methyl }} \mathrm{st} & 9.8 & 2955 & 5.7 & \\ v_{39} & 2992 & 2992 & \mathrm{CH}_{\text {methyl }} \mathrm{st} & 9.8 & & & \\ v_{40} & 3031 & 3024 & \mathrm{CH}_{\text {rings }} \mathrm{st} & 9.0 & & & \\ v_{41} & 3059 & 3043 & \mathrm{CH}_{\text {rings }} \mathrm{st} & 11.6 & & & \\ v^{5} & & & & & 3069 & 14.5 & -0.4 \\ v_{42} & 3077 & 3077 & \mathrm{CH}_{\text {rings }} \mathrm{st} & 13.1 & & & \\ v_{43} & 3093 & 3092 & \mathrm{CH}_{\text {rings }} \mathrm{st} & 13.8 & 3118 & 13.1 & -0.3\end{array}$

${ }^{a}$ Superscripts denote modes occuring at the phase transition. ${ }^{b}$ See Ref. $14 .{ }^{c}$ Abbreviations: tor, torsion; b, bending; st, stretching; sci, scissor; rock, rocking. ${ }^{d}$ The pressure coefficients were determined by fitting the data with second order polynomials of the form $v(p)=p_{o}+a p+b p^{2}$. ${ }^{e}$ Frequencies are given at $2.3 \mathrm{GPa}$; namely, for ASA-III. 
TABLE 2: Pressure effects on Raman modes of ASA-II single crystal

\begin{tabular}{|c|c|c|c|c|}
\hline \multirow[b]{2}{*}{$\operatorname{mode}^{a}$} & \multicolumn{2}{|r|}{$1 \mathrm{~atm}}$. & \multicolumn{2}{|c|}{$\begin{array}{c}\mathrm{d} v / \mathrm{dp}^{c} \\
\left(\mathrm{~cm}^{-1} \mathrm{GPa}^{-1}\right)\end{array}$} \\
\hline & freq $\left(\mathrm{cm}^{-1}\right)$ & tentative assignment $^{b}$ & $a$ & $b$ \\
\hline$f_{1}$ & 47 & & 6.2 & -0.4 \\
\hline$f_{2}$ & 59 & & 8.8 & -0.6 \\
\hline$f_{3}$ & 61 & & 10.2 & -0.7 \\
\hline$f_{4}$ & 69 & & 12.4 & -0.6 \\
\hline$f_{5}$ & 106 & $\mathrm{CH}_{3}$ tor $+\mathrm{Ph}-\mathrm{COOH}$ tor & 13.1 & -0.6 \\
\hline$f_{6}$ & 118 & $\mathrm{O} \cdots \mathrm{O} \mathrm{st}+\mathrm{CH}_{3}$ tor & 13.8 & -0.4 \\
\hline$f_{7}$ & 130 & $\mathrm{CH}_{3}$ tor & 18.4 & -1.3 \\
\hline$f^{1}(2.5)$ & 131 & & 8.5 & -0.3 \\
\hline$f_{8}$ & 173 & $\mathrm{Ph}-\mathrm{COOH} b$ & 14.0 & -0.3 \\
\hline$f_{9}$ & 264 & $\mathrm{Ph}-\mathrm{COOH} \mathrm{b}$ & 5.3 & -0.1 \\
\hline$f_{10}$ & 291 & $\mathrm{Ph}-\mathrm{OCOCH}_{3} \mathrm{~b}$ & 5.0 & 0.0 \\
\hline$f^{2}(9.1)$ & 292 & & & \\
\hline$f^{3}(5.8)$ & 314 & & & \\
\hline$f_{11}$ & 324 & $\mathrm{C}-\mathrm{C}(\mathrm{COOH})-\mathrm{C} b$ & 3.9 & -0.1 \\
\hline$f_{12}$ & 328 & & 4.8 & -0.4 \\
\hline$f_{13}$ & 383 & $\mathrm{OCOCH}_{3}$ rock. b $+\mathrm{COOH}$ rock. $b$ & 3.6 & -0.2 \\
\hline$f_{14}$ & 426 & $\mathrm{O}-\mathrm{CO}-\mathrm{CH}_{3} \mathrm{sci} \mathrm{b}+\mathrm{CC}_{\text {rings }} \mathrm{b}$ & 6.0 & -0.3 \\
\hline$f_{15}$ & 439 & $\mathrm{OCOCH}_{3} \mathrm{~b}+\mathrm{CC}_{\text {rings }} \mathrm{b}$ & 2.4 & 0.1 \\
\hline$f_{16}$ & 515 & & 0.6 & 0.1 \\
\hline$f_{17}$ & 545 & & 2.8 & -0.1 \\
\hline$f_{18}$ & 555 & $\mathrm{CC}_{\text {rings }} \mathrm{b}+\mathrm{CO}-\mathrm{CH}_{3} \mathrm{sci} b$ & 3.4 & -0.1 \\
\hline$f^{4}(0.5)$ & 601 & & 0.3 & 0.0 \\
\hline$f_{19}$ & 641 & & 1.3 & 0.0 \\
\hline$f_{20}$ & 644 & & 1.8 & 0.0 \\
\hline$f_{21}$ & 668 & $\mathrm{CC}_{\text {rings }} \mathrm{b}+\mathrm{O}-\mathrm{C}=\mathrm{O} \mathrm{ib}+\mathrm{COOH}$ sci $\mathrm{b}$ & 3.8 & -0.2 \\
\hline$f_{22}$ & 706 & $\mathrm{CH}_{\text {rings }} \mathrm{b}+\mathrm{CC}_{\text {rings }} \mathrm{b}$ & 3.2 & -0.2 \\
\hline$f_{23}$ & 752 & $\mathrm{CH}_{\text {rings }} \mathrm{b}$ & 2.3 & -0.1 \\
\hline$f_{24}$ & 786 & $\mathrm{CH}_{\text {rings }} \mathrm{b}+\mathrm{COOH} b$ & 4.5 & -0.2 \\
\hline$f_{25}$ & 809 & & 3.7 & -0.2 \\
\hline$f_{26}$ & 838 & $\mathrm{CH}_{\text {rings }} \mathrm{b}$ & 3.1 & -0.1 \\
\hline$f_{27}$ & 886 & & 4.8 & -0.2 \\
\hline$f^{5}(4.4)$ & 888 & & & \\
\hline$f_{28}$ & 921 & $\mathrm{CC}_{\text {rings }} \mathrm{b}+\mathrm{O}-\mathrm{CO}-\mathrm{CH}_{3} \mathrm{~b}$ & 4.0 & -0.1 \\
\hline$f_{29}$ & 973 & & 4.5 & 0.0 \\
\hline$f_{30}$ & 1000 & & 4.4 & -0.2 \\
\hline$f_{31}$ & 1016 & $\mathrm{O}-\mathrm{CO}-\mathrm{CH}_{3} \mathrm{~b}+\mathrm{CH}_{3} \mathrm{~b}$ & 3.7 & -0.1 \\
\hline$f_{32}$ & 1033 & & 3.3 & 0.0 \\
\hline$f_{33}$ & 1045 & sym.rings breathing $+\mathrm{CH}_{\text {rings }} \mathrm{b}$ & 2.6 & 0.0 \\
\hline$f_{34}$ & 1139 & $\mathrm{CH}_{\text {rings }} \mathrm{b}$ & 2.2 & -0.1 \\
\hline$f_{35}$ & 1154 & $\mathrm{CH}_{\text {rings }} \mathrm{b}$ & & \\
\hline$f_{36}$ & 1195 & $\mathrm{O}-\mathrm{CO}-\mathrm{CH}_{3} \mathrm{st}+\mathrm{CH}_{3} \mathrm{~b}$ & 5.0 & -0.1 \\
\hline$f^{6}(3.4)$ & 1201 & & & \\
\hline
\end{tabular}




$\begin{array}{lclcc}f_{37} & 1223 & \mathrm{Ph}-\mathrm{OCOCH}_{3} \mathrm{st}+\mathrm{CH}_{\text {rings }} \mathrm{b} & 6.8 & -0.3 \\ f_{38} & 1247 & & & \\ f_{39} & 1260 & \mathrm{CH}_{\text {rings }} \mathrm{b} & 2.2 & -0.1 \\ f_{40} & 1557 & & 4.3 & 0.0 \\ f_{41} & 1576 & \mathrm{CC}_{\text {rings }} \mathrm{st} & 4.4 & -0.1 \\ f_{42} & 1608 & \mathrm{CC}_{\text {rings }} \mathrm{st} & 5.3 & -0.1 \\ f_{43} & 1630 & \mathrm{C}=\mathrm{O} \text { carbox. st }+\mathrm{OH} \mathrm{b} & 0.9 & 0.2 \\ f_{44} & 1752 & \mathrm{C}=\mathrm{O} \text { ester st } & 0.4 & 0.2 \\ f_{45} & 2943 & \mathrm{CH}_{\text {methyl }} \mathrm{st} & 8.6 & -0.1 \\ f^{7}(3.4) & 2956 & & & \\ f_{46} & 2992 & \mathrm{CH}_{\text {methyl }} \mathrm{st} & 9.8 & -0.2 \\ f_{47} & 3026 & \mathrm{CH}_{\text {rings }} \mathrm{st} & 10.9 & -0.2 \\ f_{48} & 3062 & \mathrm{CH}_{\text {rings }} \mathrm{st} & 9.0 & 0.8 \\ f_{49} & 3080 & \mathrm{CH}_{\text {rings }} \mathrm{st} & 15.0 & -0.4 \\ f_{50} & 3094 & \mathrm{CH}_{\text {rings }} \mathrm{st} & 14.6 & -0.4 \\ f^{8}(5.1) & 3137 & & & \\ f^{9}(5.1) & 3151 & & & \end{array}$

${ }^{a}$ The modes present at ambient conditions are denoted with subscripts.

Peaks/shoulders not present at ambient conditions are denoted with a superscript and the pressure (in GPa) at which they appear is shown in parenthesis. ${ }^{b}$ See Ref. 14. ${ }^{c}$ The pressure coefficients were determined by fitting the data with second order polynomials of the form $v(p)=p_{o}+a p+b p^{2}$. 